\title{
Collaborations interprofessionnelles autour d'un projet d'innovation pédagogique à l'université : un même ethos pour de nouvelles interactions
}

\author{
Interprofessional collaborations around an educational \\ innovation project at the university: \\ the same ethos for new interactions
}

\section{Colaboraciones interprofesionales en torno a un proyecto de innovación pedagógica en la universidad: el mismo ethos para nuevas interacciones}

\author{
Emmanuel Brandl, ingénieur de recherche \\ École nationale supérieure des sciences de l'information et des bibliothèques (Enssib), France \\ emmanuel.brandl@enssib.fr
}

\begin{abstract}
RÉSUMÉ
La mise en place d'un projet d'innovation pédagogique par le numérique porté par le service commun de la documentation (SCD) d'une université (désignée " $L R$ » dans l'enquête) a été l'occasion de mener une enquête exploratoire sur les conditions de mise en place de collaborations interprofessionnelles entre enseignants-chercheurs (EC) et bibliothécaires. Notre question de départ consistait à se demander pourquoi des $E C$ réputés très occupés étaient en capacité de s'investir dans un projet collaboratif par définition incertain et chronophage. Nous avons émis l'hypothèse qu'il fallait reconstituer les schèmes de perception et d'appréciation (Bourdieu, 1992) de leur métier d'enseignant-chercheur, car ils sont au principe de la logique de leurs investissements professionnels. Hypothèse heuristique : si l'investissement des EC dans la collaboration prend sens, c'est parce qu'ils partagent un même "ethos professionnel " (Fusulier, 2011) qui les porte à déployer des stratégies professionnelles collaboratives. La présentation des résultats revient d'abord sur les conditions socioprofessionnelles de production de ces schèmes et de cet ethos, avant d'en décrire le contenu et la corrélation que l'on établit entre ethos et collaboration interprofessionnelle.
\end{abstract}


Mots-clés : collaborations interprofessionnelles, interaction collaborative, innovation pédagogique, numérique, ethos professionnel, trajectoire socioprofessionnelle, stratégies, dispositions socioprofessionnelles

\section{ABSTRACT}

The implementation of a digital pedagogical innovation project led by the library of a University (designated "LR" in the survey) was the opportunity to conduct an exploratory survey on the conditions for implementing interprofessional collaborations between lecturer-researchers and librarians. We wondered why lecturer-researchers deeply involved in their research projects, their teaching responsibilities, and their administrative work could get involved in a collaborative project by definition uncertain and time-consuming. We formulated the hypothesis that we had to reconstruct the schemes of perception and appreciation (Bourdieu, 1992) of their profession because this is where the logic of their professional investments lies. Heuristic hypothesis: the lecturer-researchers' commitment to collaborating takes on meaning as they share the same "professional ethos" (Fusulier, 2011), leading them to implement collaborative professional strategies. The results' presentation first returns to the socioprofessional conditions on this ethos's production before describing its content and the correlation between ethos and interprofessional collaboration.

Keywords: interprofessional collaborations, collaborative interaction, pedagogical innovation, digital, professional ethos, socio-professional trajectory, strategies, socio-professional dispositions

\section{RESUMEN}

La implementación de un proyecto de innovación pedagógica digital dirigido por la biblioteca de una Universidad (designado "LR" en la encuesta) permitió realizar una encuesta exploratoria sobre las condiciones para establecer colaboraciones interprofesionales entre profesores-investigadores $(\mathrm{Pl})$ y bibliotecarios. Sin embargo, los $\mathrm{PI}$ se consideran muy ocupados por sus proyectos de investigación, su carga docente y administrativa. Nuestra cuestión inicial consistía en preguntarse la razón por la cual dichos profesores e investigadores eran capaces de involucrarse en un proyecto colaborativo por definición incierto y cronófago. Planteamos la hipótesis de la necesidad de reconstituir los esquemas de percepción y de apreciación (Bourdieu, 1992) de su profesión, que guían la lógica de los compromisos profesionales. Hipótesis heurística: si la involucración de los PI en la colaboración toma sentido es porque los PI comparten entre ellos (y con los bibliotecarios) el mismo "ethos profesional" (Fusulier, 2011), que les lleva a desarrollar estrategias profesionales colaborativas. La presentación de los resultados considera, en primer lugar, las condiciones socioprofesionales de producción de este ethos, y luego se describe su contenido y la correlación que hacemos entre ethos y colaboración interprofesional.

Palabras clave: colaboraciones profesionales, interacción colaborativa, innovación pedagógica, digital, ética profesional, disposiciones socioprofesionales 


\section{Enjeu et cadrage de l'étude}

Si la question des collaborations interprofessionnelles à l'université « ne fait guère partie de la culture dominante ni des "allant de soi" institutionnels », c'est d'abord parce que «l'interdisciplinarité (...) remet en question la structure bureaucratique des universités » (Kosremelli Asmar et Wacheux, 2007, p. 7; Caruso et Rhoten, 2001). Pourtant, il apparaît incontestable aujourd'hui que les outils et dispositifs numériques participent d'une radicale transformation des modèles d'apprentissage traditionnels et suscitent de nombreuses réflexions, en particulier quant à l'évolution pédagogique de l'université. Au cœur de ces réflexions se trouve précisément la question des formes de collaboration qui doivent être (re)déployées au sein des établissements universitaires. C'est ainsi que « les établissements se doivent de relever le défi de la mise en œuvre d'une nouvelle culture de la collaboration » (Paivandi, 2016, p. 63).

Les enjeux des conditions de collaboration déployées (ou non) entre équipes pédagogiques et service commun de la documentation (SCD) à l'université autour d'un projet d'innovation pédagogique par le numérique tels qu'ils sont analysés ici paraissent bien réels : c'est une question contemporaine qui travaille en profondeur les universités françaises.

\section{Cadrage théorique}

Les termes "collaborations " et "collaborations interprofessionnelles " seront ici retenus. Les collaborations établies ou à venir autour du projet dont il est question, porté par un service de la documentation (SCD) au sein d'une université française (LR), et qui sera présenté plus bas, seront abordées comme autant de façons de travailler, de s'organiser et de fonctionner collectivement autour d'un projet unique, pour réaliser un travail suivant des objectifs communs (Kosremelli Asmar et Wacheux, 2007 , p. 9). La collaboration interprofessionnelle ayant en effet pour caractéristique minimale, comme dans le cas présent, de réunir " des membres d'au moins deux groupes professionnels autour d'un but commun » (Robidoux et al., 2007, p. 12), par exemple, la lutte contre la désaffection des étudiants de premier cycle universitaire.

L'analyse sociologique des collaborations professionnelles ou interprofessionnelles est relativement rare. Cette problématique reste plutôt le fait des sciences de l'information et de la communication, des sciences de gestion ou du management, mais aussi de la philosophie (d'Amour, 1997, 2003). Elle est particulièrement développée dans le champ de la santé (Couturier et Belzile, 2018) et, plus précisément pour les hôpitaux, dans l'objectif d'améliorer les services aux patients (Kosremelli Asmar et Wacheux, 2007), mais également dans le champ de l'éducation, cependant plutôt orientée vers les questions plus strictement pédagogiques (Lameul et Loisy, 2014), vers les usages du numérique pour l'apprentissage (Barbot et Massou, 2011; Massou et Lavielle-Gutnik, 2017) et sur la question des relations enseignantsétudiants (Paivandi et Espinosa, 2013).

Pourtant, la question des collaborations interprofessionnelles des enseignants-chercheurs (EC) mérite une attention sociologique particulière. II s'agit de comprendre pourquoi des EC s'investissent dans des projets de collaborations interprofessionnelles alors que, rationnellement, tout semble indiquer qu'ils ont intérêt à ne pas le faire. Quelle est donc la logique des investissements individuels et collectifs dans des collaborations interprofessionnelles autour d'un projet marqué par le fait :

1) De n'avoir aucun caractère obligatoire?

2) D'être, de par son aspect « innovant », incertain et chronophage, d'abord parce que l'innovation remet en question les " pratiques conventionnelles " établies et met à mal les logiques organisationnelles qui participent d'une bonne coordination des actions (Becker, 1988, p. 301), et 
ce, alors même qu'au-delà de perceptions et d'actions différentes de chaque activité selon la discipline (Boyer et Coridian, 2002), les EC sont globalement caractérisés par le fait d'être déjà très occupés par leurs projets de recherche, leurs charges d'enseignement et diverses charges (d'aucuns diront « surcharges ») administratives (Faure, Soulié et Millet, 2005)?

3) De porter en outre sur l'enseignement, aspect historiquement et structurellement dévalorisé du métier d'enseignant-chercheur (EC) et sans impact sur une quelconque évolution de carrière? II apparait ainsi que le " tropisme savant » du monde universitaire conduit à rejeter culturellement les questions pédagogiques du côté des activités primaires et peu dignes d'intérêt (Millet, 2013).

4) D'aller enfin à l'encontre de valeurs structurelles qui viennent caractériser ce " groupe professionnel " (Dubar, 2003) telles que l'autonomie et l'« individualisme pédagogique " (Paivandi, 2016, p. 24), lesquelles participent d'une " absence de culture de la collaboration » (p. 24) dans les activités d'enseignement?

C'est à cet aspect pour le moins paradoxal que nous souhaitons apporter des éléments de compréhension. L'argument le plus évident est celui de la réussite étudiante : malgré les difficultés, les EC se mobilisent pour collaborer autour de la lutte contre la désaffection des étudiants à l'université; c'est d'ailleurs l'objet déclaré du projet d'innovation pédagogique par le numérique auquel ces $E C$ ont participé (voir plus bas). Mais on ne fait que déplacer la question : pourquoi ces EC se mobilisent-ils pour lutter contre la désaffection des étudiants (au risque de mettre à mal leur carrière)? Pourquoi s'orientent-ils vers ce choix professionnel somme toute risqué? Quel intérêt ont-ils à s'investir dans un tel projet? En outre, tout EC ne s'investit pas dans un tel projet : la distribution des EC qui s'investissent dans de tels projets se fait-elle au hasard (des occasions, des rencontres, etc.)? En réalité, c'est à une question de " posture » d'EC, ou mieux, c'est à la question des modes de perception et d'appréciation de la fonction d'EC à l'université que ces interrogations revoient.

Pour tenter de répondre à ces interrogations, nous avons posé comme hypothèse que l'analyse des dispositions socioprofessionnelles des individus est en elle-même pertinente pour comprendre le choix que ces professionnels font de s'investir dans des collaborations interprofessionnelles autour d'un projet d'innovation pédagogique par le numérique. Ces « dispositions », entendues comme structures cognitives individuelles qui orientent, voire déterminent les actions dans un contexte donné, sont incorporées au long d'un processus de socialisation professionnelle, lui-même défini comme processus de "socialisation secondaire "(Berger et Luckmann, 2018), lequel participe de "l'intériorisation de sous-mondes institutionnels spécialisés (valeurs, normes, règles) construits en référence à un champ spécialisé d'activités » (Dubar, 1991, p. 102). Elles fonctionnent alors comme des schèmes de perception et d'action qui structurent la vision que les EC ont de l'« espace des possibles " (Bourdieu, 1992) professionnel qui s'offre à eux, et par là, la logique de leurs orientations et investissements professionnels, ou dit autrement, de leurs stratégies professionnelles, si l'on entend cette notion au sens d'" actions qui sont raisonnables sans être le produit d'un dessein raisonné ou, à plus forte raison, d'un calcul rationnel » (Bourdieu, 1980, p. 85-86).

\section{Cadrage méthodologique}

L'enquête que nous avons réalisée repose principalement sur une série de 20 entretiens semi-directifs libres d'une heure et demie à deux heures, menés en face à face, par téléphone (Cachia et Millward, 2011) et visioconférence (Lo lacono, Symonds et Brown, 2016). Ces entretiens ont été menés avec des enseignants-chercheurs ( $n=15: 3$ informatique, 3 biologie, 2 mathématiques, 2 électronique, 2 physique, 2 sciences de l'éducation-informatique, 1 histoire) et des bibliothécaires $(n=5)$. Les deux guides d'entretien (EC et bibliothécaires) ont été élaborés afin de permettre de mener une analyse comparative. Nous 
n'exploiterons ici que la partie consacrée aux enseignants-chercheurs. Pour les besoins de la démonstration, il sera cependant fait mention à certains moments des bibliothécaires de l'enquête.

Le choix de ce type d'entretiens tient à ce qu'il s'agissait moins de recueillir la totalité de la vie d'un individu que de contextualiser le récit pour focaliser l'attention sur la trajectoire sociale en lien avec le groupe professionnel auquel l'individu estime appartenir et la pratique professionnelle à laquelle il estime participer (Bertaux, 1997). Il s'agissait de recueillir des « récits de pratiques contextualisées » (Bertaux, 1997, p. 65), car il ne s'agit pas d'extraire d'un récit toutes les significations qu'il contient, mais seulement celles qui sont pertinentes pour l'objet de la recherche et qui y prennent le statut d'indices. Pour mener les entretiens, nous avons alors utilisé la méthode « biographique », méthode qui consiste à intégrer un individu dans les processus collectifs en lui faisant évoquer, décrire, réfléchir sur des périodes de sa vie. Le canevas d'entretien était construit en deux temps intriqués, que l'analyse thématique a méthodologiquement permis de distinguer :

- Un temps consacré à l'activité professionnelle d'enseignant-chercheur. Nous avons ainsi recueilli des indicateurs de positions (avoir suivi telle formation initiale, avoir exercé tel métier, avoir obtenu tel poste, un poste à tel moment, dans telle université, avoir aujourd'hui telles activités, les limites rencontrées, etc.) et de prises de position (les raisons évoquées pour justifier les choix professionnels, le sens donné à ses activités, les accords, les désaccords, les valeurs associées, etc.).

- Un temps consacré au projet lui-même (indicateurs privilégiant les prises de position) : prise de connaissance et perception des contenus, intérêt exprimé ou non pour le projet, raisons évoquées autour de la participation ou non, forme des collaborations, mais aussi actions effectivement réalisées, etc.

Nous avons ensuite cherché à mettre en relation ces deux aspects, en intégrant l'un (le projet) dans l'autre (l'activité professionnelle) pour en analyser la signification. Pour ce faire, nous avons d'abord procédé à une analyse thématique des entretiens (Combessie, 2001). Cette technique procède d'une lecture attentive des entretiens pour en repérer les mots clés qui permettent de répondre à la question : de quoi parle ce passage? Nous avons ainsi identifié des unités thématiques dont une part était déjà proposée dans le canevas d'entretien. Chaque unité thématique était précisément documentée par des sousthèmes. Par exemple, pour le thème " activité professionnelle ", on aura " enseignement ", et pour " enseignement », on pouvait avoir " responsabilité pédagogique », "MCF », " informatique », etc. Pour le thème " projet », on aura " intégré des chapitres d'ebooks », " collaboration "... Dans un deuxième temps, l'analyse transversale (Demazière et Dubar, 1997, p. 98) permet de procéder à une analyse des systèmes d'opposition entre différentes détermination/signification. Un tableau à double entrée permet d'identifier des systèmes d'association et d'opposition :

- « biologie » (comme discipline) est « associé à » ou « opposé à » " transversalité »;

- " sciences humaines " et " plateforme pédagogique " sont " associées à " ou " opposées à » " réticences d'ordre culturel », « peur du plagiat »;

- « MCF » (ou « PR ») est « associé à » ou « opposé à » " responsabilité pédagogique »;

- « responsabilité pédagogique » (comme sous-thème) est lui-même " associé à » ou " opposé à » " être un bon pédagogue » - " ça me ralentit dans mon avancée de carrière »;

- " filières professionnalisantes » ou " sciences dures " sont " associées à " ou " opposées à " " utilisent davantage les outils collaboratifs »;

- etc. 
Enfin, l'analyse comparative des entretiens permet de mettre en évidence les récurrences discursives entre données de position et de prise de position.

Ce travail effectué, les récurrences permettent d'opérer des regroupements et donc de dégager des groupes de position et de prise de position. Ce travail d'interprétation procède nécessairement d'une forme de simplification de la réalité étudiée, toujours plus complexe, car les pratiques sociales et professionnelles sont le fait de mélanges et de bricolages subtils. L'objectif de l'analyse reste de démêler l'écheveau de la réalité afin d'en mettre en exergue certaines prévalences, d'en expliciter et ainsi d'en mieux comprendre les contextes étudiés.

Ainsi, quant aux positions (et conditions) socioprofessionnelles, les récurrences constatées ont permis de retenir trois variables empiriques qui conditionnent les prises de position constatées : "discipline d'enseignement ", " compétences numériques » et " articulation enseignement-recherche ». Quant aux prises de position et aux valeurs, l'analyse comparative a permis de construire cinq variables : " rénovation » (des métiers, de la pédagogie), « transversalité », " éthique de la conviction », " disposition à l'adaptation » et « valeurs de désintéressement ». Ces cinq variables participent de la constitution de ce que l'on a appelé un " ethos professionnel ». Au final, aucun enseignant-chercheur n'enferme à lui seul l'ensemble des caractéristiques de cet ethos. Ce dernier, tel qu'il est décrit ici, correspond à un ethos « idéal-typique » (Weber, 1971) et résulte de fait d'une construction intellectuelle nécessairement arbitraire à certains égards (Mauger et Fossé-Poliak, 1983).

Les données d'entretien ont été complétées par une série d'observations dites " in vivo " (Laplantine, 1996), lesquelles consistent à intégrer par moment des espaces professionnels afin d'en observer les comportements contextualisés. Nous avons notamment participé à une journée d'étude sur l'innovation pédagogique organisée conjointement par la Direction des usages du numérique et le SCD de l'université concernée.

L'enquête est donc de type monographique et qualitative. Au regard du nombre limité d'entretiens que nous avons menés, il faut évidemment faire preuve de prudence quant au degré de généralisation de nos résultats. Dire cependant qu'on ne peut généraliser les résultats d'enquêtes monographiques au-delà des cas étudiés, c'est « confondre la généralisation empirique des résultats avec la généralisation théorique d'un schème d'analyse ou d'un modèle explicatif qui a été construit à propos d'un cas empirique et concret » (Champagne, 1990, p. 37). C'est pourquoi il apparaît scientifiquement légitime d'avancer que le modèle d'analyse et d'interprétation des données proposé ici peut être transposable à d'autres contextes universitaires et permettre d'envisager une démarche comparative.

\section{Analyse des résultats}

\section{Éléments de contexte : un projet d'innovation pédagogique par le numérique}

En 2016, l'appel à manifestation d'intérêt (AMI) lancé par le ministère de l'Éducation nationale, de l'Enseignement supérieur et de la Recherche (MENESR) de France pour la thématique «Production / éditorialisation de ressources pédagogiques et accompagnement de leurs usages " a retenu un projet porté par le service commun de la documentation (SCD) de l'Université LR intitulé « Des ebooks pour la Licence ».

L'intérêt du projet résidait dans le fait que l'offre d'ebooks pour le cycle Licence était partielle, alors même que l'objectif du projet consistait à s'appuyer sur les atouts réels ou supposés de la documentation électronique (" accès nomade, disponibilité immédiate, égalité entre les sites de l'Université ») pour lutter 
contre l'échec des étudiants de première année de Licence, en déployant dans la durée " une offre d'ebooks à destination des étudiants de Licence, en cohérence avec les enseignements ». Pour ce faire, il avait alors été décidé « de mettre en place sur l'ensemble de l'année 2017 une offre multidisciplinaire à destination des Licences ».

Un des objectifs affichés du projet était d'y associer le plus systématiquement possible les équipes pédagogiques. C'est ainsi que la pertinence des offres éditoriales d'ebooks et la qualité des plateformes de lecture (accès Web mobiles, utilisabilité, contenus) étaient caractérisées " en lien avec les communautés enseignantes ». Les pratiques pédagogiques des EC paraissaient ainsi " au cœur du dispositif pour intégrer au mieux les possibilités offertes par les ebooks : tester le principe de la classe inversée, scénariser l'utilisation des ressources, favoriser l'acquisition de connaissances et savoir-faire ciblés, développer l'autonomie de l'apprenant " (tous les extraits sont tirés du cahier des charges du projet).

Il était donc entendu que le SCD possède l'expertise de l'analyse documentaire et technique du marché des offres numériques (des " bouquets »), quand les EC sont experts de leur discipline et sont invités à intervenir dans les choix de contenus et les tests pédagogiques des ressources. Le projet intègre ainsi théoriquement un principe de complémentarité et une logique de collaboration.

\section{Variables de position et prises de position vis-à-vis de l'innovation pédagogique par le numérique}

Il est indéniable que des facteurs tels que le format (des ebooks pédagogiques homothétiques) ou encore le pilotage du projet participent pour leur part des conditions de collaboration, puisqu'elles déterminent les conditions de réception et d'appropriation du projet par les EC. Toutefois, ces facteurs organisationnels n'agissent pas d'eux-mêmes. La réception, la compréhension des attentes formulées et la décision de s'investir dans une collaboration sont comme " filtrées " par les schèmes de perception et d'appréciation que les EC font du projet en fonction de leur "profil ", lequel se rapporte à leur trajectoire socioprofessionnelle. Nous ne développerons ici que cet aspect de l'enquête.

Quatre variables des trajectoires socioprofessionnelles sont apparues constitutives des positions professionnelles déterminantes des prises de position des EC pour la mise en place d'interactions collaboratives avec les bibliothécaires autour de la question de l'innovation pédagogique par le numérique : la discipline d'enseignement, la pratique pédagogique, les compétences numériques et la place faite à l'enseignement et à la recherche dans l'activité professionnelle. Ces variables " font système ", elles sont toutes nécessaires, mais non suffisantes et participent de la constitution d'un ethos professionnel.

\section{DISCIPLINE D’ENSEIGNEMENT ET PRATIQUE PÉDAGOGIQUE}

Si l'offre de contenus était destinée à l'ensemble des EC de l'Université LR, toutes disciplines confondues, il s'avère que seuls les EC des disciplines que l'on nommera ici schématiquement " scientifiques et techniques » (biologie, mathématiques, informatique, sciences de l'éducation, gestion...) ont peu ou prou répondu à l'appel. Comme l'analyse des entretiens a permis de l'identifier, il y a un effet de la discipline d'enseignement sur les prises de position vis-à-vis du projet. C'est que la discipline d'appartenance détermine le rapport à la pratique collaborative. II ressort globalement de cela que les EC des disciplines scientifiques et techniques perçoivent les EC des disciplines littéraires comme moins enclins à la pratique pédagogique collaborative, donc moins à même de participer à des projets de collaboration autour des questions pédagogiques. 
« J'avais commencé à le dire à une table ronde mais j'ai pas insisté, j'ai aussi ensuite analysé les données du travail collaboratif à l'université. Là, c'était rigolo, parce qu'on s'aperçoit, et c'est déjà reconnu ailleurs, que les littéraires travaillent moins de manière collaborative que les scientifiques. Les filières professionnalisantes (santé et compagnie) utilisent aussi davantage les outils numériques collaboratifs. " (JS, MCF, informatique).

La socialisation disciplinaire détermine également les modes de perception et d'appréciation que l'EC aura de son propre cours. Du côté des disciplines scientifiques et techniques, les contenus mêmes qui sont à transmettre (et à assimiler), tels les schémas, figures, vocabulaires..., définissent un travail de transmission du savoir basé sur une question d'assimilation de connaissances précises et contrôlées, et moins sur un savoir exploratoire appuyé sur une démarche personnelle de documentation (Millet, 2000). C'est une des raisons pour lesquelles les filières littéraire, artistique et SHS se caractérisent par une tendance à la frilosité quand il s'agit de déposer les supports de cours sur les plateformes pédagogiques, principalement de peur du plagiat. C'est qu'ici, à la différence des disciplines aux savoirs plus standardisés, les EC ont tendance à composer leurs propres cours, faisant de ceux-ci en quelque sorte une œuvre originale, une production intellectuelle inédite qui appartient en propre à son producteur, car pensée comme une production originale et unique. On pourrait appeler ça un " sens de la propriété intellectuelle ».

« Alors oui, c'est vrai, il y a une incitation à utiliser ce mode d'information, de discussion et de contact avec les étudiants [plateforme pédagogique]. Néanmoins, il y a une espèce de plafond de verre qui fait qu'en sciences humaines et sociales, sans doute en lettres aussi, on hésite à déposer autre chose qu'un plan de cours... Moi, je suis à l'interface des sciences plus dures et des sciences plus molles, donc c'est un peu plus facile. Mais si je porte un regard sur mes collègues de sciences humaines, les collègues historiens par exemple, ne déposent ici essentiellement que des plans de cours, et pas de cours qui seraient formalisés. [Pourquoi à votre avis?] Je ne peux pas répondre pour eux, je ne sais pas, je pense qu'il y a ces réticences d'ordre culturel sur la propriété et la peur du plagiat et la question des droits d'auteur, c'est une vraie question sensible en sciences humaines, beaucoup plus qu'avec mes collègues en sciences dures » (MC, MCF, Histoire Médiévale).

De surcroit, dans le cadre du projet, l'intégration des ebooks au contenu des cours sur la plateforme pédagogique était associée au fait d'élaborer un « scénario pédagogique ». Or, l'élaboration d'un scénario pédagogique impose de pouvoir se projeter dans le temps. Mais il s'avère que le rapport au temps de la pratique pédagogique est variable selon la discipline : les disciplines scientifiques et techniques participent d'une anticipation pédagogique qui est plus délicate à appréhender pour les disciplines « littéraires » telles que les sciences humaines et sociales et les filières lettres, arts et langues. La raison de cette variation est à chercher dans la nature même du savoir disciplinaire :

«Parce qu'elle porte sur des corpus de connaissances existants et finis (x chapitres de y pages dans $z$ matières...), la pratique d'apprentissage (en médecine - ndr) peut faire l'objet d'une programmation et d'un découpage temporel relativement plus précis (un chapitre toutes les deux heures, par demi-journée>>>) que s'il s'agissait d'un travail de fabrication intellectuelle, moins réitératif et plus génératif, dans le cadre duquel les contours et les contenus de la connaissance, nécessairement plus flous et plus vagues, sont aussi moins délimités et délimitables. » (Millet, 2000, p. 386)

La standardisation des connaissances et des documents pédagogiques facilite et même rend possible le travail d'anticipation pédagogique. 


\section{LES COMPÉTENCES NUMÉRIQUES}

II ressort de l'analyse des entretiens que l'innovation pédagogique par le numérique se définit de deux manières différentes : soit comme une manière de créer de nouveaux outils d'apprentissage - ludification dynamique, mise à disposition documentaire via une plateforme pédagogique... -, soit comme une façon de faciliter le travail quotidien de l'enseignant par la notion d'automatisation (par exemple des corrigés d'exercices), laquelle est associée à la notion d'autonomisation (de l'étudiant).

L'innovation pédagogique ne se réduit pas, tant s'en faut, à l'usage du numérique. Mais dans ce spectre des possibles, le choix de l'EC vers l'une ou l'autre de ces prises de position ou vers le cumul de ces dernières sera orienté par ses compétences numériques. Elles viennent déterminer la place (au sens d'usage et de légitimité) prêtée au numérique dans les questions d'innovation pédagogique et s'accordent avec une certaine perception des fonctions du numérique en pédagogie : soit le numérique est perçu comme un outil de rationalisation du travail enseignant, soit il participe d'une vision quelque peu « enchantée » de l'innovation technologique en pédagogie.

Or, les motivations à participer à un travail collaboratif autour d'un projet d'innovation pédagogique en dépendent. En effet, plus la compétence numérique individuelle est élevée, plus l'intérêt du numérique pour l'innovation pédagogique est probant (plus l'usage du numérique est perçu comme légitime). Le numérique prend alors la forme d'outils d'apprentissage et son intérêt réside dans la ludification de l'enseignement qu'il permet.

De plus, une des particularités d'un projet cherchant à innover, et ce, d'autant plus que les technologies à maitriser sont elles-mêmes innovantes, tient en ce qu'il doit inventer une partie au moins des structures sociales et professionnelles sur lesquelles il s'appuie : transversalité, compétences individuelles nouvelles, etc. Or, cela est d'autant plus ardu que les conventions sont inscrites dans le matériel et les équipements de production (Becker, 1988). Car il faut inventer le matériel, mais aussi les compétences nécessaires à la maitrise de ces nouveaux outils. Or, l'apprentissage le plus souvent autodidacte inhérent à l'innovation induit des contraintes temporelles fortes : les EC les plus aguerris interviewés passent le plus clair de leur temps professionnel, mais aussi et surtout personnel, à l'exploration et à l'exploitation de ces outils pédagogiques numériques en testant un nombre substantiel de plateformes et de logiciels.

« [Et pour les phases tests des plateformes?] Ça, finalement, je le fais sur mon temps libre, c'est presque un hobby de tester ces plateformes, de programmer des petits trucs, donc ça me prend du temps de loisir. » (SH, MCF, informatique)

II ne s'agit pas tant d'investir du temps dans la préparation des cours que dans la maitrise, la conception et le déploiement d'outils numériques à visée pédagogique. On comprend alors de surcroît qu'un EC sera d'autant plus frileux à l'investissement dans un tel projet qu'il maitrise moins les compétences numériques nécessaires à la maitrise des outils eux-mêmes. D'autant que ce temps d'appropriation et de production se situe hors des systèmes de mérite et constitue une sorte de travail invisible (Losego, 2004). Soulignons qu'à l'inverse, l'offre étant limitée à des ebooks homothétiques, les EC aux compétences numériques avérées se sont révélés également frileux à l'usage de ces ebooks dans le cadre d'une démarche d'innovation pédagogique, ceux-ci n'apparaissant que comme un prolongement dématérialisé du support papier.

En substance, on constate que l'usage des ressources pédagogiques numériques ne se distribue pas au hasard, mais qu'il s'inscrit dans des trajectoires socioprofessionnelles d'usages. La notion de « trajectoire d'usage » étant empruntée à Serge Proulx (2002). 


\section{L'ARTICULATION ENSEIGNEMENT-RECHERCHE}

Les collaborations interprofessionnelles envisagées lors du projet portent sur les activités d'enseignement des EC. II faut donc que les EC se saisissent des questions d'enseignement... La remarque pourrait paraître triviale, voire déplacée si l'on ignorait que la caractéristique des statuts de maitre de conférences et de professeur des universités - à la différence relative des PRAG (professeurs agrégés) par exemple tient en ce que leur activité professionnelle s'articule autour de l'enseignement et de la recherche. II s'agit donc d'une double activité (sans même parler des charges administratives) qui se pratique dans des proportions variables. Cela signifie que l'identité professionnelle des EC est elle-même double, faite d'une identité professionnelle de chercheur et d'une identité professionnelle d'enseignant, chacune répondant à des enjeux professionnels propres. Or, à l'université, l'avancement dans la carrière d'un EC repose moins sur l'excellence de ses activités d'enseignement que sur l'excellence de ses activités de recherche. Rappelons à ce titre qu'en France, si les EC bénéficient d'une formation à la recherche, il n'en va pas de même de l'enseignement (Ménard, Hoffmann et Lameul, 2017).

«Faire travailler des enseignants-chercheurs sur la partie pédagogique, c'est un grand challenge, parce que le système français et le système mondial disent que vous êtes un bon maitre de conférences et un bon professeur des universités si vous êtes un bon chercheur : la pédagogie n'a pas de valeur, à mon grand regret (...). » (FA, MCF, électronique)

Or, ce qui rassemble les EC aux statuts de maitre de conférences et de professeur des universités qui ont peu ou prou répondu à l'appel du porteur de projet, c'est qu'ils sont tout d'abord enseignants avant d'être chercheurs, conservant malgré leur ancienneté des responsabilités pédagogiques et déplaçant le champ de leurs recherches académiques initiales vers des problématiques relatives à la pédagogie, notamment en problématisant les notions d'innovation pédagogique et d'outils numériques pour l'enseignement : ils participent d'une conversion de leur investissement pédagogique en intérêt scientifique. La valeur cardinale autour de laquelle se retrouvent ces EC se situe dans l'importance qu'ils accordent à l'enseignement dans leur métier d'EC et, par là, à l'intérêt qu'ils portent aux transformations de leur écosystème professionnel d'enseignant, notamment quant aux profils d'apprenant des étudiants de Licence lié à un sentiment de besoin d'adaptation des outils d'apprentissage, notamment par le numérique, afin de rendre l'étudiant plus « actif » de son processus d'apprentissage.

Ce facteur « enseignement » est déterminant, car il conditionne l'intérêt qu'un EC pourra porter à un projet d'innovation pédagogique, le temps qu'il consentira à y consacrer et donc aux formes de collaborations qu'il sera possible d'envisager. Au-delà des limites "techniques" soulevées (discipline, compétences numériques, pratique pédagogique), et probablement avant même que les EC ne connaissent précisément le contenu de ce type de projet, celui-ci a toutes les chances de rencontrer spontanément un écho favorable chez ces EC. En effet, il participe de la valorisation des compétences pédagogiques investies par ces derniers dans un contexte universitaire qui relativise précisément ces compétences.

\section{Un ethos professionnel}

L'ethos correspond à un ensemble de valeurs, de principes, de manières de se percevoir et d'agir dans une société ou un groupe professionnel donné sans nécessairement faire l'objet d'une pleine et entière conscience de cet ethos. L'ethos professionnel est une manière de se percevoir et d'agir " en qualité de ", ici : d'EC (et de bibliothécaire). Il est générateur des prises de position professionnelles et participe ainsi d'une identité de métier, si l'on considère que celle-ci se définit dans les modes de perception et d'appréciation de son métier à l'université et dans le sens que ces acteurs donnent à leurs activités professionnelles (Marsollier, 1999). 
Au cœur de l'ethos professionnel se pose la question du rapport entre « vouloir agir » et " pouvoir (d')agir » « comme professionnel » (Jorro, 2009, p. 3). Dès lors, « l'ethos professionnel peut être générateur d'une critique ou d'un malaise à l'égard d'un cadre organisationnel ou situationnel dans lequel la pratique se déroule » (Fusulier, 2011, p. 105), et porte les acteurs concernés vers des négociations, des transactions, des actions (collectives) visant à modifier ou à adapter leur écosystème organisationnel. C'est précisément ce que l'on constate.

\section{RÉNOVATION DES MÉTIERS ET TRANSVERSALITÉ}

Le premier point commun qu'il faut relever, et qui recouvre un ensemble de valeurs et dispositions à l'action, est celui d'une stratégie de rénovation - débanalisation des métiers. En se saisissant de l'innovation et du numérique pédagogique, les EC qui contribuent au projet participent en effet d'une stratégie de rénovation et de re-légitimation de l'enseignement à l'université, du métier et de la carrière d'enseignant plutôt que de celle de chercheur. Ces professionnels vont se saisir des opportunités qui leurs sont offertes pour rénover leur métier en s'emparant de ce qui, contextuellement, permet d'innover ou de produire un effet d'innovation, comme ici le « numérique », l'« innovation pédagogique » et l'« innovation pédagogique par le numérique ».

« J'avais des élèves en scientifique, je me suis dit "faut vraiment faire des figures pour qu'ils comprennent ce qui se passe sans qu'on fasse des maths", ce qui me met en désaccord avec des collègues. J'ai des collègues de génération 0.0 (à qui) j'ai montré ça récemment, et ils m'ont encore engueulé en disant "mais enfin, M., qui c'est qui t'a dit de faire des maths?" J'ai dit que je faisais des maths descriptives, ça n'existe pas des maths descriptives, j'ai dit "écoute, c'est dans la maquette des L1 géoscience: "les mathématiques seront descriptives"'", c'est-à-dire qu'on démontre pas les trucs, on manipule, on comprend les choses, etc. Donc, du coup, on a un projet avec D. T. qui était à la réunion l'autre jour, où on a réussi à se faire acheter une quinzaine de tablettes par l'université, et du coup, lui sur les équa diff et moi en géoscience, on va faire manipuler les élèves sur les tablettes, pour faire des exos directement. Donc on va concevoir un nouveau type d'exercices, j'ai déjà fait ça sous Google, et je l'ai testé avec mes M2F, ça a été déstabilisant pour eux. C'est intéressant, ça. » (YM, PR, mathématiques)

D'un point de vue organisationnel, la collaboration inter-professionnelle, mais en réalité tout comme la collaboration intra-professionnelle, fait partie intégrante de ces opportunités qu'il faut savoir saisir afin de rénover son activité, en débanalisant et en actualisant le métier exercé. II ne s'agit plus de rester " enfermé » seul dans sa pratique, mais de s'inscrire dans un écosystème professionnel contemporain plus large et plus complexe qui favorise un élargissement du champ d'intervention et des compétences maitrisées, ce qui amène notamment ces EC à transgresser un certain état de l'organisation ou de la division du travail à l'Université LR. À ce titre, une des notions qui structure les différents discours de ces EC est celle de la «transversalité ».

Cette entreprise de rénovation du métier se caractérise ici par le fait de s'inscrire dans des logiques de fonctionnement qui participent d'une forme d'avant-garde organisationnelle en proposant de mettre en œuvre « de nouvelles combinaisons entre les différentes ressources de l'entreprise » (Alter, 1993) dans laquelle EC (et bibliothécaires) s'inscrivent, et ce, en l'absence de procédures combinatoires standardisées. Pour ce faire, il faut trouver des sources de collaboration et des raisons de collaborer; le projet livrera l'opportunité de le faire. 


\section{RÉNOVATION DE LA PÉDAGOGIE ET ÉTHIQUE DE LA CONVICTION}

Dans cette entreprise de rénovation, bibliothécaires et EC se retrouvent autour d'une même conception de la "pédagogie». Ce qui caractérise les EC de l'étude, c'est qu'ils partagent un même ethos d'enseignant : quitte à mettre à mal leur carrière, ils adoptent tous une attitude militante dans le principe même d'être enseignant avant d'être chercheur, et cela, dans un écosystème qui valorise pourtant toujours la recherche (« on appelle ça la foi je crois », MCF, électronique) :

« Moi, je serai jamais hors classe (signifiant par-là « classe exceptionnelle »-ndr) parce que j'ai pas fait ce qu'il fallait, je suis trop dans mes trucs, c'est pas grave, et puis si je publie, je publie sur des trucs de didactique, c'est pas considéré comme de la recherche (...). » (YM, PR, mathématiques)

Les activités qu'ils développent autour de la notion d'innovation pédagogique, avec par exemple la ludification numérique des exercices, mais aussi l'usage d'" escapes games » (MG, PRAG, biologie), sont caractérisées par le fait de prendre leurs distances avec les formes les plus académiques de l'enseignement: pour valoriser l'enseignement à l'Université LR, il faut rénover cet enseignement, " moderniser » l'enseignement à l'université, c'est-à-dire les méthodes pédagogiques. Ici, EC et bibliothécaires partagent un même « rejet des pédagogies dites de la transmission (la magistralité), pour favoriser l'activité des étudiants, ce qui correspond au glissement souvent évoqué du paradigme de l'enseignement au paradigme de l'apprentissage » (Alter, 1993). L'enseignant se meut ici en " coach » ou en « guide » qui apprécie peu le cours magistral, lui préférant les contextes de plus grande proximité (TD, TP) et une documentation pédagogique plus proche de l'accompagnement que de l'enseignement.

« Le prof qui fait un cours magistral, pour moi, ça n'a pas beaucoup de sens, je déteste ça. Je préfère les avoir en TD, en TP (...). L'idée c'est de les voir deux fois au premier semestre pour leur mettre un peu la pression aussi. Et puis suivre un peu avec eux leurs difficultés, que ce soient des difficultés sociales, psychologiques, matérielles, pouvoir les orienter vers les services de l'Université aussi qui peuvent les aider à gérer certains problèmes (...). C'est un peu du coaching. (...) Je prépare beaucoup en amont mes séances et pendant les séances, ce que je fais, c'est que je les observe, je les coache. » (MG, MCF, biologie)

Au fond, ces EC partagent entre eux (et avec les bibliothécaires du projet) une même " éthique de la conviction » : ils partagent en effet la même conviction des vertus pédagogiques des technologies du numérique (relativement à leur perception du rôle du numérique dans l'innovation pédagogique, laquelle doit quelque chose à leur niveau de compétence en la matière). Malgré l'absence de preuves tangibles de sa pertinence pédagogique comme de sa capacité à créer de la motivation (Garcia, 2003), et qu'il soit réellement mis en œuvre ou pas, le numérique se révèle à leurs yeux un moteur d'apprentissage plus efficace que l'encadrement institutionnel et, de ce fait, une solution viable au problème du désistement étudiant.

\section{UNE MÊME « DISPOSITION À L’ADAPTATION »}

Ce qui est également partagé par l'ensemble des acteurs interviewés, EC et bibliothécaires confondus, c'est qu'ils sont tous, à un degré ou à un autre, comme conduits par l'idée d'un changement nécessaire, induisant qu'il est de facto nécessaire d'adapter et de s'adapter au contexte local et sociétal actuel, à un nouvel état de la société et de l'université à l'échelle mondiale. L'université est perçue comme caractérisée par le déploiement des technologies du numérique et l'arrivée d'un public d'étudiants sinon "nouveau » d'un point de vue sociodémographique, du moins "nouveau » dans ses usages, ses comportements et sa formation : temps de concentration moindre, relative démotivation, désintérêt pour les formes les plus " scolaires » de l'enseignement, usage du numérique, déconnexion avec les outils pédagogiques les plus 
classiques... Ils partagent ainsi ce que Denis Lemaître appelle le " schème de l'adaptation », sorte d'état d'esprit qui participe d'une conviction progressiste auquel est associée une vision praxéologique de l'activité professionnelle, c'est-à-dire une activité « orientées vers l'action et l'efficacité (...) et dans le but de toujours proposer des améliorations » (Lemaître, 2018, p. 2).

Même si les preuves qui permettraient de fonder l'intérêt et la pertinence aussi généralisée du numérique dans la réussite des étudiants (comme des élèves) viennent à manquer, l'innovation pédagogique par le numérique, à l'appui d'une association forte, voulue et instrumentalisée (Garcia, 2003), entre numérique et innovation, participe de fait de ces facteurs d'adaptation. La problématique est identique : s'adapter pour lutter contre les risques de déclassement professionnel et ainsi "garder la valeur sociale positive " (Goffman, 1974) de sa position professionnelle et de son institution d'appartenance, en élaborant de nouvelles légitimités professionnelles (ici à travers le numérique). Ces acteurs partagent donc une même conception pragmatique et adaptative de leur activité professionnelle.

\section{DES VALEURS DE DÉSINTÉRESSEMENT PARTAGÉES}

L'analyse montre que ces deux corps professionnels partagent enfin l'idée d'une profession venant affirmer des valeurs de désintéressement, via une pratique plus orientée vers l'autre que vers soi. Pour les enseignants-chercheurs, alors que la recherche reste un élément primordial de la formation, de l'identification au métier comme de l'évolution de la carrière, l'enseignement et la réussite étudiante, voire les charges administratives passent devant les gratifications individuelles de la recherche académique (c'est la valeur cardinale de leur ethos professionnel).

« II y a une double vision qui s'affronte : une vision très personnelle et un peu nombriliste, c'està-dire mon nom sur la publi, quel rang, combien de publications, etc., et de l'autre côté, pour moi, y a la construction de la nation, y a la formation de la jeunesse pour son devenir (...). C'est un positionnement dans la nation et dans la société : je me suis positionné non pas en tant qu'individuel mais plus sur "ça a fonctionné pour moi et j'aimerais bien que ça continue à fonctionner pour les autres et pour les jeunes qui arrivent dans l'enseignement supérieur". » (EF, MCF, électronique)

Ces professionnels placent tous les notions de "service public», de service "au » public, de " démocratisation de l'accès » (à l'enseignement, aux documents et plus globalement au savoir), mais aussi de diffusion (des connaissances, de la documentation), de "réussite " (des étudiants), de " dévouement», de "changement», de "transversalité » (intra et inter professionnelle), de "décloisonnement» au cœur de leurs activités professionnelles respectives. Ces professionnels participent d'un « monde » social de conventions, de principes et de justifications cohérent qu'ils partagent (sans le savoir vraiment), leur permettant de s'entendre pour coordonner leurs activités lorsque l'occasion leur en est donnée.

\section{Ethos professionnel et collaborations interprofessionnelles}

Le principe de l'ethos professionnel est à rechercher dans la combinaison de deux facteurs : la position et la pente de la trajectoire professionnelle.

La position occupée dans un espace social de légitimités et de hiérarchies professionnelles se détermine par la possession d'un certain « capital » (Bourdieu, 1979). Pour les EC, les positions professionnelles articulent "capital recherche » (publications, financements...) et "capital pédagogique " (entendu au sens large, englobant méthode d'enseignement, responsabilités, projets...), la distribution de ces capitaux définissant la position et la trajectoire (carrière) professionnelles. Ici, les EC (quel que soit leur statut: 
maitres de conférences, professeurs des universités ou PRAG) ont tous un "capital pédagogique » supérieur à leur "capital recherche ». Or, si, comme l'a montré J.-C. Passeron (1991), les EC sont davantage reconnus pour leur contribution personnelle au développement d'un savoir que pour la qualité de leurs activités d'enseignement, lesquelles relèvent d'une activité perçue comme primaire, faite de tâches subalternes, alors la structure du capital professionnel des EC de l'enquête contribue à ce qu'ils occupent des positions professionnelles plutôt situées au bas de la hiérarchie des légitimités universitaires. À ce titre, l'investissement des EC de cette enquête dans les questions pédagogiques (ils sont d'abord investis dans cet aspect de leur métier), comme nous l'avons vu, participe du sentiment d'avoir « tiré un trait sur leur carrière » :

« Donc j'ai mis cette contrainte-là (être un bon pédagogue), ça me ralentit dans mon avancée de carrière. Au CNU, j'ai fait le même constat : la pédagogie, c'est aux oubliettes, ça ne rapporte rien. » (FA, MCF, électronique)

In fine, les trajectoires socioprofessionnelles sont quant à elles marquées par une courbe ascendante. Les EC sont tous entrés tardivement à l'université et sont d'abord passés par un enseignement dans le primaire ou le secondaire (général ou professionnel) durant plusieurs années. Ils sont par-là formés aux techniques d'enseignement du secondaire et s'appuient d'ailleurs parfois sur la documentation de " collègues » du secondaire pour constituer leurs cours ou leurs parcours (de ludification par exemple). Cette formation initiale est constitutive de leur trajectoire socioprofessionnelle et de leur socialisation professionnelle, et participe dès lors de la perception de ce que doit être à leurs yeux l'enseignement à l'université.

L'ethos professionnel participe alors d'un processus dynamique : il produit un cercle vertueux qui crée de la motivation à collaborer de façon intra et inter professionnelle: tout à la fois "francs-tireurs " organisationnels, "militants » et professionnels " hétérodoxes », EC (et bibliothécaires) sont ici les opérateurs du changement universitaire. Ils participent d'une lutte symbolique pour la transformation de l'enseignement à l'Université LR parce qu'ils sont « objectivement » voués à être " portés » par la même chose et prêts à collaborer pour cette même chose : la rénovation des métiers (les EC parlent parfois de " dépoussiérer » les pratiques pédagogiques) et par-là des positions professionnelles, par l'innovation, ici pédagogique, adossée au " numérique ». Ils participent ainsi d'une logique de renversement de la table des valeurs académiques pour modifier les règles du jeu définissant l'ordre des carrières universitaires en faisant du stigmate pédagogique un emblème.

II s'agit de lutter contre des principes (de classement), des signes (distinctifs) et un certain monopole (le monopole de la définition légitime de ce qu'est être EC) pour créer un nouvel ordre plus favorable à leur position et à leur trajectoire professionnelle. L'innovation pédagogique autorise l'espoir d'une « remise en question de certains rapports de force et de certaines croyances jusqu'alors instituées » (Lemieux, 2007, p. 192) et d'inventer de nouveaux dispositifs organisationnels et techniques permettant à terme de contraindre différemment l'ordre actuel des collaborations socioprofessionnelles à l'université. Dans cette lutte, pour une cause qui n'est autre que la reconnaissance d'une identité de métier en laquelle ils croient et qu'ils portent, située au-delà des cloisonnements professionnels, les professionnels enquêtés (EC et bibliothécaires) apparaissent comme des alliés objectifs dont la collaboration peut permettre d'augmenter à la fois leurs convictions et leur impact transformateur.

\section{Conclusion}

L'article a permis de revenir sur les conditions de production des dispositions socioprofessionnelles, produit de trajectoires socioprofessionnelles données qui structurent la logique des orientations et des investissements professionnels. Ces dispositions sont partagées, font système et participent de ce que 
l'on a désigné, après Bernard Fusulier (2011), d'un ethos professionnel. Cet ethos est sous-jacent et préalable aux interactions propres aux collaborations: il oriente les schèmes de perception et d'appréciation des dynamiques professionnelles vers des collaborations interprofessionnelles, lesquelles sont placées au service d'une revalorisation des positions professionnelles.

Si la notion de "collaboration interprofessionnelle " a pour caractéristique minimale de réunir des membres d'au moins deux groupes professionnels autour d'un but commun, au vu des résultats de l'enquête, ce « but commun " se révèle être double. Si les EC mettent bien le numérique pédagogique au service de la cause des étudiants de Licence en vue de lutter contre leur désaffection, ils le mettent inséparablement au service de leurs propres trajectoires socioprofessionnelles. Cette double dynamique partagée, celle de la "visée externe » (objectifs) et celle de la "motivation interne » (stratégies), prédispose ces EC à entrer dans une série d'interactions collaboratives. On pourrait dire qu'en la matière s'assemblent (pour collaborer) ceux qui, professionnellement, se ressemblent.

\section{Liste de références}

Alter, N. (1993). Innovation et organisation : deux légitimités en concurrence. Revue française de sociologie. 1993, 34(2), 175-197. https://www.persee.fr/doc/rfsoc 0035-2969 1993 num 3424240

Barbot, M.-J. et Massou, L. (dir.). (2011). TIC et métiers de l'enseignement supérieur. Émergences, transformations, PUN Éditions universitaires de Lorraine.

Becker, H. S. (1988). Les mondes de l'art. Flammarion.

Berger, P. et Luckmann, T. (2018). La construction sociale de la réalité. Armand Colin.

Bertaux, D. (1997). Les récits de vie. Nathan.

Bourdieu, P. (1979). La distinction. Critique sociale du jugement de goût. Minuit.

Bourdieu, P. (1980). Le sens pratique. Minuit.

Bourdieu, P. (1992). Réponses : pour une anthropologie réflexive, Seuil.

Boyer, R. et Coridian, C. (2002). Transmission des savoirs disciplinaires dans l'enseignement universitaire: une comparaison histoire/sociologie. Sociétés contemporaines. 48, 41-61.

Cachia, M. et Millward, L. (2011). The telephone medium and semi-structured interviews: a complementary fit. Qualitative Research in Organizations and Management: An International Journal, 6(3). 265-277.

Caruso D et Rhoten D. (2001, avril). Lead, follow, get out of the way: sidestepping the barriers to effective practice of interdisciplinarity. A new mechanism for knowledge production and re-integration in the age of information. The Hybrid Vigor Institute.

Champagne, P. (1990). Faire l'opinion. Minuit.

Combessie, J-C. (2001). La méthode en sociologie. La Découverte. Repères.

Couturier, Y. et Belzile, L. (2018). La collaboration interprofessionnelle en santé et services sociaux. Les Presses de l'Université de Montréal.

D’Amour, D. (1997). Structuration de la collaboration inter-professionnelle dans les services de santé de première ligne au Québec [thèse de doctorat, Université de Montréal]. https://www.collectionscanada.gc.ca/obj/s4/f2/dsk2/tape17/PQDD_0003/NQ32608.pdf

D'Amour, D. (dir.). (2003). Étude comparée de la collaboration interorganisationnelle et de ses effets : le cas des services en périnatalité. Canadian Health Services Research Foundation.

Demazière, D. et Dubar, C. (1997). Analyser les entretiens biographiques (l'exemple des récits d'insertion). Nathan.

Dubar, C. (1991). La socialisation. Construction des identités sociales et professionnelles. Armand Colin.

Dubar, C. (2003). Sociologie des groupes professionnels en France : un bilan prospectif. Dans P.-M. Menger (dir.). Les professions et leurs sociologies. Modèles théoriques, catégorisations, évolutions. MSH, 51-60.

https://doi.org/10.4000/books.editionsmsh.5715 
Faure, S. Soulié, C. et Millet, M. (juin 2005). Enquête exploratoire sur le travail des enseignants chercheurs. Vers un bouleversement de la " table des valeurs académiques »? [Rapport]. https://doi.org/10.13140/RG.2.1.3644.1766

Fusulier, B. (2011). Le concept d'ethos. Recherches sociologiques et anthropologiques. 42(1), 97-109. https://doi.org/10.4000/rsa.661

Garcia, S. (2003). Croyance pédagogique et innovation technologique. Le marché de la formation à distance au service de la « démocratisation » de l'enseignement supérieur. Actes de la recherche en sciences sociales, 149, 42-60.

Goffman, E. (1974). Les rites d'interaction. Minuit.

Jorro, A. (2009). La construction de l'ethos professionnel en formation alternée. Travail et apprentissage, 3, 13-25.

Kosremelli Asmar, M. et Wacheux, F. (2007). Facteurs influençant la collaboration inter-professionnelle : cas d'un hôpital universitaire, Université Saint-Joseph. Conférence Internationale en Management. Beyrouth. Liban. https://halshs.archives-ouvertes.fr/halshs-00170357

Lameul, G. et Loisy, C. (dir.). (2014). La pédagogie universitaire à l'heure du numérique. Questionnement et éclairage de la recherche. De Boeck.

Laplantine, F. (1996). La description ethnographique. Nathan.

Lemaître, D. (2018). L'innovation pédagogique en question : analyse des discours de praticiens. Revue internationale de pédagogie de l'enseignement supérieur, 34(1). https://doi.org/10.4000/ripes.1262

Lemieux, C. (2007). À quoi sert l'analyse des controverses? Société d'études soréliennes. Mil neuf cent, 1(25), p. 191-212. https://doi.org/10.3917/mnc.025.0191

Lo lacono, V. Symonds, P. et Brown, D. H. K. (2016). Skype as a Tool for Qualitative Research Interviews. Sociological Research Online, 21(2), 103-117.

Losego, Ph. (2004). Le travail invisible à l'université : le cas des antennes universitaires. Sociologie du travail, 46(2)., 187204.

Marsollier, Ch. (1999). Innovation pédagogique et identité professionnelle : le concept de « rapport à l'innovation ». Recherche \& Formation, 31, 11-29. https://doi.org/10.3406/refor.1999.1564

Massou, L. et Lavielle-Gutnik, N. (2017). Enseigner à l'université avec le numérique - Savoirs, ressources, médiations. De Boeck supérieur.

Mauger, G. et Fossé-Poliak, C. (1983). Les loubards. Actes de la recherche en sciences sociales, 50, 49-67.

Ménard, L. Hoffmann, C. et Lameul, G. (2017). Effets de la formation à l'enseignement sur les pratiques des nouveaux enseignants-chercheurs. Recherche et formation, 84, 125-140.

Millet, M. (2000, mai). Les étudiants de médecine et de sociologie à l'étude. Matrices disciplinaires, nature des savoirs, et pratiques intellectuelles : une analyse sociologique comparée des logiques sociales et cognitives du travail étudiant [thèse de doctorat, Université Lumière - Lyon 2]. http://theses.univ-lyon2.fr/documents/lyon2/2000/mmillet

Millet, M. (2013). Les cadres cognitifs de la socialisation. Savoirs, apprentissages et scolarisation. Habilitation à diriger des recherches. Université de Poitiers.

Paivandi, S. (dir). (2016, juin). Articulation et collaboration entre les équipes pédagogiques et les services de documentation au cœur de la transformation pédagogique de l'enseignement supérieur. Rapport du LISEC (Laboratoire interuniversitaire des sciences de l'éducation et de la communication).

Paivandi, S. et Espinosa, G. (2013). Les TIC et la relation entre enseignants et étudiants à l'université. Distances et médiations des savoirs, 4. https://doi.org/10.4000/dms.425

Passeron, J. C. (1991). Le raisonnement sociologique. L'espace non poppérien du raisonnement naturel. Nathan.

Proulx, S. (2002). Trajectoires d'usages des technologies de communication : les formes d'appropriation d'une culture numérique comme enjeu d'une société du savoir. Annales des télécommunications. 57(3-4), 180-189.

Robidoux, M., Barrette, S. Mathieu S. et Thomas L. (2007, septembre). Cadre de référence. Collaboration interprofessionnelle. École en chantier - Oser construire ensemble. Université de Sherbrooke.

Weber, M. (1971). Économie et Société, tome 1. Plon. 